M. Colţoiu and V. Vâjâitu

Nagoya Math. J.

Vol. 157 (2000), 1-13

\title{
LOCALLY TRIVIAL FIBRATIONS WITH SINGULAR 1-DIMENSIONAL STEIN FIBER OVER $q$-COMPLETE SPACES
}

\author{
MIHNEA COLŢ,OIU AND VIOREL VÂÂATU
}

\begin{abstract}
In connection with Serre's problem, we consider a locally trivial analytic fibration $\pi: E \longrightarrow B$ of complex spaces with typical fiber $X$. We show that if $X$ is a Stein curve and $B$ is $q$-complete, then $E$ is $q$-complete.
\end{abstract}

\section{$\S 1$. Introduction}

Let $\pi: E \rightarrow B$ be a locally trivial analytic fibration of complex spaces with Stein fiber $X$ of dimension $n$.

The following question was raised by Serre [17]:

Under the above assumptions, does it follow that $E$ is Stein if $B$ is Stein?

The answer is 'Yes' for $n=0$ (Stein [23] and Le Barz [12]) and $n=1$ (Mok [13]. In fact, some partial results were previously proved by various authors, Siu [19], Sibony [18], Hirschowitz [9], etc).

However, for $n \geq 2$ there are counterexamples to Serre's question (see Skoda [21], Demailly [7], and Coeuré-Loeb [3]).

Related to this circle of ideas we study the case when the base $B$ is $q$-complete. The normalization is chosen such that Stein spaces correspond to 1-complete spaces.

For $n=0$, i.e., $E$ is a topological covering of $B$, Ballico [2] proved the $q$-completeness of $E$. This is a particular case of a result due to Vâjâitu [24] which gives that if $\pi: Y \rightarrow Z$ is a locally trivial analytic fibration with hyperconvex fibre and $Z$ is $q$-complete, then $Y$ is $q$-complete. (A complex space $S$ is said to be hyperconvex if $S$ is Stein and has a negative exhaustion function which is continuous and plurisubharmonic.)

For $n=1$, in order to generalize Mok's result, Vâjâitu [26] showed if $X$ is non-singular, $E$ is $q$-complete if $B$ is $q$-complete. It remained the open problem when $X$ is a singular Stein curve.

Received June 26, 1998. 
Our main result gives a complete answer to this question. It can be stated as follows.

THEOREM 1. Let $\pi: E \rightarrow B$ be a locally trivial analytic fibration with typical fiber $X$. If $X$ is a Stein curve and $B$ is q-complete, then $E$ is qcomplete, too.

We remark that for $q=1$ the above theorem can be deduced from the case when the fiber is non-singular. This is due to the fact that the class of Stein spaces is invariant under finite holomorphic surjections (see Narasimhan [14]).

When $q>1$ the situation is drastically different because it is not known (see Colţoiu [6]) if the following holds:

Let $p: Y \rightarrow Z$ be a finite surjective holomorphic map of complex spaces. Assume $Y$ is q-complete. Does it follow that $Z$ is q-complete?

(When $Z$ is $q$-complete, then $Y$ is $q$-complete. See Vâjâitu [25].)

To avoid this difficulty we use essentially an approximated extension of $q$-convex functions defined on complex subspaces with control of the directions of positivity of the extended function. Also the quasi-plurisubharmonic functions (Peternell [15]; see also Demailly [8]) will play an important rôle in the proof.

\section{$\S 2$. Preliminaries}

Throughout this paper all complex spaces are assumed to be reduced and with countable topology.

Let $Y$ be a complex space and $T_{y} Y$ denotes the (Zariski) tangent space of $Y$ at $y$. Set $T Y=\cup_{y \in Y} T_{y} Y$.

A subset $\mathcal{M} \subset T Y$ is said to be a linear set over $Y$ (of codimension $\leq q-1)$ if for every point $y \in Y, \mathcal{M}_{y}:=\mathcal{M} \cap T_{y} Y \subset T_{y} Y$ is a complex vector subspace (of codimension $\leq q-1$ ). If $W \subset Y$ is an open subset, we have an obvious definition of the restriction $\mathcal{M}_{\left.\right|_{W}}$.

Let $\pi: Z \rightarrow Y$ be an analytic morphism of complex spaces and $\mathcal{M}$ a linear set over $Y$. For every $z \in Z$ we have an induced C-linear map of complex vector spaces $\pi_{*, z}: T_{z} Z \rightarrow T_{y} Y$, where $y=\pi(z)$. We set

$$
\pi^{*} \mathcal{M}:=\bigcup_{z \in Z}\left(\pi_{*, z}\right)^{-1}\left(\mathcal{M}_{y}\right)
$$


Clearly, $\pi^{*} \mathcal{M}$ is a linear set over $Z$. Moreover, if codim $(\mathcal{M}) \leq q-1$, then $\operatorname{codim}\left(\pi^{*} \mathcal{M}\right) \leq q-1$.

A (local) chart of $Y$ at a point $y \in Y$ is a holomorphic embedding $\iota: U \rightarrow \widehat{U}$, where $U \ni y$ is an open subset of $Y$ and $\widehat{U}$ is an open subset of some euclidean space $\mathbf{C}^{n}$. Holomorphic embedding means that $\iota(U)$ is an analytic subset of $\widehat{U}$ and the induced map $\iota: U \rightarrow \iota(U)$ is biholomorphic.

Suppose $\iota: U \rightarrow \widehat{U}$ is a local chart at $y$; then the differential map $\iota_{*, y}: T_{y} Y \rightarrow \mathbf{C}^{n}$ of $\iota$ at $y$ is an injective homomorphism of complex vector spaces.

Let $D \subset \mathbf{C}^{n}$ be an open subset. A function $\varphi \in C^{\infty}(D, \mathbf{R})$ is said to be $q$-convex if the quadratic form

$$
L(\varphi, z)(\xi)=\sum_{i, j=1}^{n} \frac{\partial^{2} \varphi}{\partial z_{i} \partial \bar{z}_{j}}(z) \xi_{i} \bar{\xi}_{j}, \xi \in \mathbf{C}^{n},
$$

has at least $n-q+1$ positive eigenvalues for every $z \in D$, or equivalently, there exists a family $\left\{M_{z}\right\}_{z \in D}$ of $(n-q+1)$-dimensional complex vector subspaces of $\mathbf{C}^{n}$ such that $L(\varphi, z)_{\left.\right|_{M_{z}}}$ is a positive definite form for all $z \in D$.

Let $Y$ be a complex space. A function $\varphi \in C^{\infty}(Y, \mathbf{R})$ is said to be $q$ convex if every point of $Y$ admits a local chart $\iota: U \rightarrow \widehat{U} \subset \mathbf{C}^{n}$ such that there is an extension $\widehat{\varphi} \in C^{\infty}(\widehat{U}, \mathbf{R})$ of $\varphi_{\left.\right|_{U}}$ which is $q$-convex on $\widehat{U}$. (This definition does not depend on the local embeddings.)

We say that $Y$ is $q$-complete if there exists a $q$-convex function $\varphi \in$ $C^{\infty}(Y, \mathbf{R})$ which is exhaustive, i.e., the sublevel sets $\{\varphi<c\}, c \in \mathbf{R}$, are relatively compact in $Y$.

The following is due to Peternell [15].

Definition 1. Let $Y$ be a complex space, $W \subset Y$ an open set, $\mathcal{M}$ a linear set over $W$, and $\varphi \in C^{\infty}(W, \mathbf{R})$.

(a) Let $y \in W$. Then we say that $\varphi$ is weakly 1 -convex with respect to $\mathcal{M}_{y}$ if there are: a local chart $\iota: U \rightarrow \widehat{U}$ of $Y$ with $y \in U \subset W, \widehat{U} \subset \mathbf{C}^{n}$ open set, and an extension $\widehat{\varphi} \in C^{\infty}(\widehat{U}, \mathbf{R})$ of $\varphi_{\left.\right|_{U}}$ such that $L(\widehat{\varphi}, \iota(y))\left(\iota_{*, y} \xi\right) \geq 0$ for every $\xi \in \mathcal{M}_{y}$.

We say that $\varphi$ is weakly 1-convex with respect to $\mathcal{M}$ if $\varphi$ is weakly 1-convex with respect to $\mathcal{M}_{y}$ for every $y \in W$.

(b) The function $\varphi$ is said to be 1-convex with respect to $\mathcal{M}$ if every point of $W$ admits an open neighborhood $U \subset W$ such that there exists a 1-convex function $\theta$ on $U$ with $\varphi-\theta$ weakly 1 -convex with respect to $\mathcal{M}_{\left.\right|_{U}}$. 
It is not difficult to see that the extension $\widehat{\varphi}$ of $\varphi$ is irrelevant for the above definition. In particular, if the functions $\varphi$ and $\psi$ are (weakly) 1convex with respect to $\mathcal{M}$, so is their $\operatorname{sum} \varphi+\psi$.

Definition 2. Let $Y$ be a complex space and $\mathcal{M}$ a linear set over $Y$. We denote by $\mathcal{B}(Y, \mathcal{M})$ the set of all $\varphi \in C^{o}(Y, \mathbf{R})$ such that every point of $Y$ admits an open neighborhood $D$ on which there are functions $f_{1}, \ldots, f_{k} \in C^{\infty}(D, \mathbf{R})$ which are 1 -convex with respect to $\mathcal{M}_{\left.\right|_{D}}$ and

$$
\varphi_{\left.\right|_{D}}=\max \left\{f_{1}, \ldots, f_{k}\right\}
$$

From [24] and [4] we quote

Proposition 1. Let $\mathcal{M}$ be a linear set over a complex space $Y$ and $\varphi \in$ $\mathcal{B}(Y, \mathcal{M})$. Then for every $\eta \in C^{o}(Y, \mathbf{R}), \eta>0$, there exists $\widetilde{\varphi} \in C^{\infty}(Y, \mathbf{R})$ which is 1-convex with respect to $\mathcal{M}$ and

$$
\varphi \leq \widetilde{\varphi}<\varphi+\eta
$$

In particular, if $\mathcal{M}$ has codimension $\leq q-1$, then $\widetilde{\varphi}$ is $q$-convex.

From [15] we have:

Proposition 2. Let $Y$ be a complex space and $\varphi \in C^{\infty}(Y, \mathbf{R})$ a $q$ convex function. Then there is a linear set $\mathcal{M}$ over $Y$ of codimension $\leq q-1$ such that $\varphi$ is 1-convex with respect to $\mathcal{M}$.

Motivated by Propositions 1 and 2, we say that a complex space $Y$ is 1-complete with respect to a linear set $\mathcal{M}$ over $Y$ if there exists an exhaustion function $\varphi \in \mathcal{B}(Y, \mathcal{M})$. Consequently a complex space $Y$ is $q$-complete if, and only if, $Y$ is 1-complete with respect to some linear set $\mathcal{M}$ of codimension $\leq q-1$.

Let us recall that a Stein space $S$ is said to be hyperconvex if there is a smooth plurisubharmonic exhaustion function $\varphi: S \rightarrow(-\infty, 0)$.

In [24] the following result has been proved:

Proposition 3. Let $\pi: E \rightarrow B$ be a locally trivial analytic fibration with hyperconvex fibre. If $B$ is 1-complete with respect to a linear set $\mathcal{M}$ over $B$, then $E$ is 1-complete with respect to $\pi^{*} \mathcal{M}$. In particular if $B$ is q-complete, then $E$ is q-complete. 
From this it follows

Corollary 1. Let $\pi: E \rightarrow B$ be a covering space with q-complete base $B$. Let $\mathcal{M}$ be a linear set over $B$ of codimension $\leq q-1$ such that $B$ is 1-complete with respect to $\mathcal{M}$. Then there exists $\mu: E \rightarrow \mathbf{R}$ a smooth exhaustion function which is 1 -convex with respect to $\pi^{*} \mathcal{M}$. In particular $E$ is q-complete.

We shall also need the following result of M. Peternell ([15], Satz 3.1).

Proposition 4. Let $Y$ be a complex space and $A \subset Y$ a closed analytic subset. Then there is a function $h \in C^{\infty}(Y, \mathbf{R})$ such that:

a) $h \geq 0,\{h=0\}=A$.

b) For every $y \in Y$ there exists an open neighborhood $U$ of $y$ and $\theta \in$ $C^{\infty}(U, \mathbf{R})$ such that

$$
\log \left(h_{\left.\right|_{U \backslash A}}\right)+\theta_{\left.\right|_{U \backslash A}}
$$

is 1-convex.

Remark 1 . The function $\log h$ is locally equal to the sum of a plurisubharmonic function and a smooth function. Such a function is called in Demailly [8] a quasi-plurisubharmonic function.

\section{$\S 3$. Construction of an auxiliary fibration}

We recall that a complex space $X$ is called hyperbolic (in the sense of Kobayashi) if the Kobayashi semidistance $d_{X}$ is a distance. See the book of S. Lang [11].

\section{Examples and properties.}

1) $\mathbf{C} \backslash\{p, q\}$ with $p, q \in \mathbf{C}, p \neq q$, is hyperbolic.

2) Any open subset of a hyperbolic space is hyperbolic.

3) Let $\pi: X^{\prime} \rightarrow X$ be a covering of complex spaces. Then $X^{\prime}$ is hyperbolic if and only if $X$ is hyperbolic.

4) Any relatively compact open subset of $\mathbf{C}^{n}$ is hyperbolic.

A proof of these facts may be found in Kobayashi [10] and Lang [11].

Let us recall also the following result. (See Siu [20], p. 176 and Royden [16], p. 311.) 
LEMMA 1. Let $F$ be a hyperbolic manifold and $W$ a connected complex space. Let $f: W \times F \rightarrow F$ be a holomorphic map such that for some $w_{o} \in W$ the restriction of $f$ to $\left\{w_{o}\right\} \times F$ is biholomorphic onto $F$. Then $f$ is independent of the variable in $W$, i.e., $f(w, x)=f\left(w_{o}, x\right)$ for all $w \in W$ and $x \in F$.

Let now $X$ be a Stein space of pure dimension 1 (Stein curve) and $W$ a connected complex space.

We assume that a biholomorphic map $\Phi: W \times X \rightarrow W \times X$ is given such that the diagram

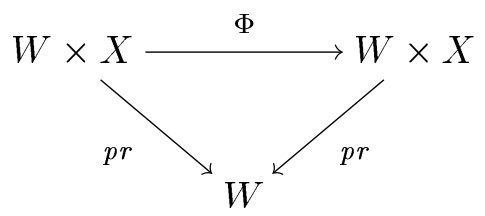

is commutative. So, for every $w \in W$ we have an automorphism of $X$, $\Phi_{w}: X \rightarrow X$, given by $\Phi_{w}(x)=\Phi(w, x)$.

Let $\nu: \widetilde{X} \rightarrow X$ be the normalization map. Every $\Phi_{w}, w \in W$, lifts to a unique automorphism $\widetilde{\Phi}_{w}$ of $\widetilde{X}$ such that the diagram

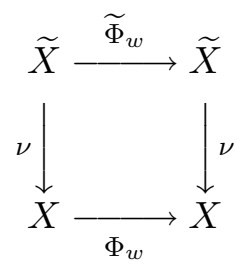

is commutative.

The maps $\left\{\widetilde{\Phi}_{w}\right\}_{w \in W}$ define a unique map $\widetilde{\Phi}: W \times \widetilde{X} \rightarrow W \times \widetilde{X}$ and we have a commutative diagram

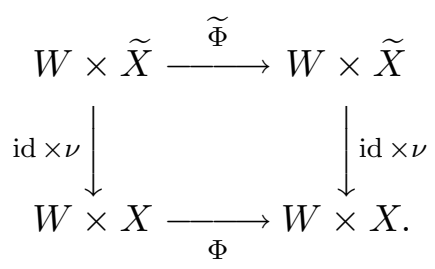

We show

LeMMA 2. $\widetilde{\Phi}$ is biholomorphic. 
This will be proved in two steps.

Step 1. $\widetilde{\Phi}$ is biholomorphic if $X$ is irreducible.

We may assume that $S:=\operatorname{Sing}(X) \neq \emptyset$. Put $\widetilde{S}=\nu^{-1}(S)$. Consider the commutative diagram of isomorphisms

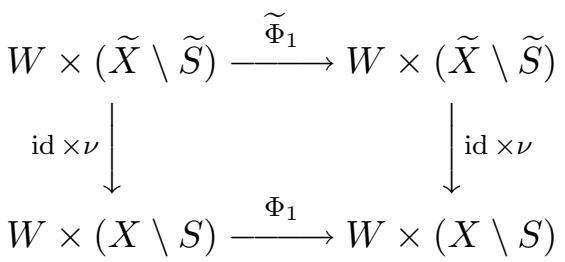

where $\Phi_{1}:=\Phi_{\left.\right|_{W \times(X \backslash S)}}$ and $\widetilde{\Phi}_{1}:=\widetilde{\Phi}_{\left.\right|_{W \times(\widetilde{X} \backslash \widetilde{S})}}$.

If $\operatorname{card}(\widetilde{S}) \geq 2$, then $\widetilde{X} \backslash \widetilde{S}$ is hyperbolic (by Examples 1), 2), and 3) in the beginning of this section). It follows from Lemma 1 that the maps $\Phi_{1}$ and $\widetilde{\Phi}_{1}$ in diagram (2) are independent of $w \in W$, therefore also the maps $\Phi$ and $\widetilde{\Phi}$ in diagram (1) are independent of $w$. In particular $\widetilde{\Phi}$ is biholomorphic.

Similarly, if $\operatorname{card}(\widetilde{S})=1$ and $\widetilde{X} \neq \mathbf{C}$, it follows that $\widetilde{X} \backslash \widetilde{S}$ is hyperbolic and $\widetilde{\Phi}$ is biholomorphic (being independent of $w \in W$ ).

It remains to study the case when $X$ has only one singular point, say $S=\left\{x_{o}\right\}$, at which $X$ is locally irreducible (therefore $\operatorname{card}(\widetilde{S})=1$ ) and $\widetilde{X}=\mathbf{C}$ is its normalization. We may assume that $\nu^{-1}\left(x_{o}\right)=0 \in \mathbf{C}$. It follows then easily that $\widetilde{\Phi}(w, \widetilde{x})=f(w) \cdot \widetilde{x}$ with $f \in \mathcal{O}^{*}(W)$, so obviously $\widetilde{\Phi}$ is biholomorphic.

Step 2. $\widetilde{\Phi}$ is biholomorphic for arbitrary 1-dimensional Stein space $X$.

Clearly we may assume that $X$ has no isolated points. Let $X=\cup X_{i}$ be the decomposition of $X$ into irreducible components. We claim first that for every index $i$ there is a unique index $j$ so that $\Phi\left(W \times X_{i}\right)=W \times X_{j}$. To show this, we let $\operatorname{Reg}(X)=\cup D_{i}$ be the decomposition into connected components with $X_{i}=\overline{D_{i}}$. Obviously, for each $i$ there is a (unique) $j$ such that $\Phi\left(W \times D_{i}\right)=W \times D_{j}$. Using the continuity of $\Phi$ the claim follows.

Now let $\widetilde{X}=\cup \widetilde{X}_{i}$ be the decomposition of $\widetilde{X}$ into connected components. Therefore $\nu\left(\widetilde{X_{i}}\right)=X_{i}$ and $\nu_{\widetilde{X}_{i}}: \widetilde{X}_{i} \rightarrow X_{i}$ is the normalization of $X_{i}$ 
From the above claim each connected component $W \times \widetilde{X}_{i}$ of $W \times \widetilde{X}$ corresponds by $\widetilde{\Phi}$ to a (unique) connected component $W \times \widetilde{X}_{j}$. Thus we have a commutative diagram

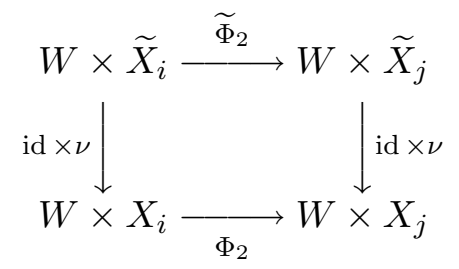

where $\Phi_{2}:=\Phi_{\left.\right|_{W \times X_{i}}}$ and $\widetilde{\Phi}_{2}:=\widetilde{\Phi}_{\left.\right|_{W \times \widetilde{X}_{i}}}$.

We fix some biholomorphic map $h: X_{j} \rightarrow X_{i}$ (e.g., $h=\Phi_{w_{o}}^{-1}$ for some $\left.w_{o} \in W\right)$ and consider the commutative diagram

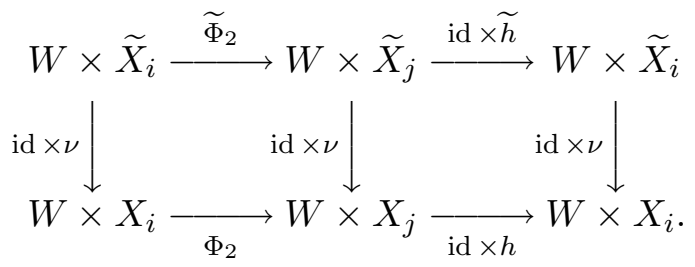

By step 1$),(\mathrm{id} \times \widetilde{h}) \circ \widetilde{\Phi}_{2}$ is biholomorphic, so $\widetilde{\Phi}_{2}$ is biholomorphic. It follows that $\widetilde{\Phi}$ is biholomorphic and the Lemma 2 is completely proved.

Lemma 3. Let $\pi: E \rightarrow B$ be a locally trivial holomorphic fibration with fibre $X$ a pure 1-dimensional Stein space.

Then there exist $\pi^{\prime}: E^{\prime} \rightarrow B$ a locally trivial holomorphic fibration with fibre $\widetilde{X}=$ the normalization of $X$ and a holomorphic map $\tau: E^{\prime} \rightarrow E$ with the following properties:

1) The diagram

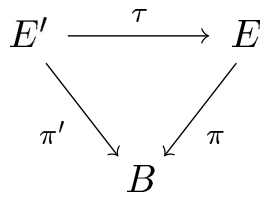

is commutative. 
2) For every $b \in B$ the induced map

$$
\tau_{b}: E_{b}^{\prime} \longrightarrow E_{b}
$$

is the normalization map $\nu: \widetilde{X} \rightarrow X$.

3) Let

$$
A:=\bigcup_{b \in B} \operatorname{Sing}\left(E_{b}\right)
$$

and $A^{\prime}:=\tau^{-1}(A)$. Then $A$ and $A^{\prime}$ are closed analytic subsets of $E$ and $E^{\prime}$ respectively and $\tau_{\left.\right|_{E^{\prime} \backslash A^{\prime}}}: E^{\prime} \backslash A^{\prime} \rightarrow E \backslash A$ is biholomorphic.

Proof. Let $\left(W_{i}\right)_{i \in I}$ be a locally finite open covering of $B$ such that $E_{\left.\right|_{W_{i}}}$ is trivial and $W_{i} \cap W_{j}$ is connected for every $i, j \in I$. We have the transition functions $\Phi_{i j}:\left(W_{i} \cap W_{j}\right) \times X \rightarrow\left(W_{i} \cap W_{j}\right) \times X$ which are biholomorphic and such that the diagram

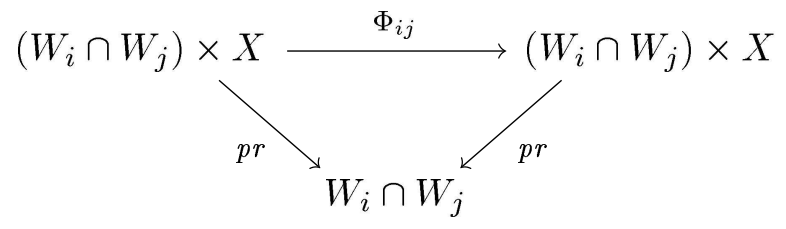

is commutative.

Therefore we have induced maps $\widetilde{\Phi}_{i j}:\left(W_{i} \cap W_{j}\right) \times \widetilde{X} \rightarrow\left(W_{i} \cap W_{j}\right) \times \widetilde{X}$ which are biholomorphisms by the previous lemma.

Then clearly $\left\{\widetilde{\Phi}_{i j}\right\}$ define the required holomorphic fibration $\pi^{\prime}: E^{\prime} \rightarrow$ $B$. All other required properties in the lemma are easily verified.

Remark 2. The above two lemmas are trivial if we assume $B$ to be normal. In this case ( $B$ normal) it is clear that $\widetilde{\Phi}_{i j}$ are biholomorphic and it is not necessary to make the assumption that the fiber $X$ is a 1-dimensional Stein space ( $X$ may be an arbitrary complex space).

\section{$\S 4$. The proof of the main result}

In this section we shall prove the subsequent Theorem $1^{\prime}$ which clearly implies Theorem 1 already mentioned in the introduction.

TheOrem 1'. Let $\pi: E \rightarrow B$ be a locally trivial analytic fibration with Stein fibre $X$ of dimension 1 and assume that $B$ is 1-complete with respect to a linear set $\mathcal{M}$ (over $B)$. Then $E$ is 1 -complete with respect to $\pi^{*} \mathcal{M}$.

In particular, $E$ is q-complete if $B$ is q-complete. 
Proof. When the fiber is non-singular Theorem $1^{\prime}$ is proved in [26].

Subsequently we deal with the singular fibre $X$.

Let $\pi^{\prime}: E^{\prime} \rightarrow B$ be a fibration with the properties stated in Lemma 3 .

Denote $p:=\pi_{\left.\right|_{A}}: A \rightarrow B$ which is a covering map. In fact $A$ can be described locally over $B$ as follows: Let $E_{\left.\right|_{U}} \simeq U \times X$ be a local trivialization. Then $A \cap \pi^{-1}(U) \simeq U \times \operatorname{Sing}(X)$ and $\operatorname{Sing}(X)$ is a discrete subset of $X$, say $\operatorname{Sing}(X)=\left\{a_{j}\right\}_{j \in J}$, since $X$ is one dimensional. By Corollary 1 there exists a smooth exhaustion function $\mu: A \rightarrow \mathbf{R}$ such that $\mu$ is 1-convex with respect to $p^{*} \mathcal{M}$.

We shall prove the following statement:

(a) Let $\eta: A \rightarrow(0, \infty)$ be any continuous function. Then there exists an open neighborhood $V$ of $A$ in $E$ and a smooth function $\widetilde{\mu}: V \rightarrow \mathbf{R}$ which is 1-convex with respect to $\left(\pi^{*} \mathcal{M}\right)_{\left.\right|_{V}}$ and

$$
\mu \leq \widetilde{\mu}<\mu+\eta
$$

on $A$.

To prove (\$) we follow an idea from (Colţoiu [5], Lemma 3); but we refine it in order to get extensions with controlled positivity directions which are necessary for our patching process.

We fix a non-negative smooth strictly subharmonic function $f: X \rightarrow \mathbf{R}$ such that $\operatorname{Sing}(X)=\{f=0\}$.

Let also $\left\{U_{i}\right\}_{i \in I}$ and $\left\{W_{i}\right\}_{i \in I}$ be locally finite open coverings of $B$ such that $U_{i} \subset \subset W_{i} \subset \subset B$ and $E$ is trivial near $\bar{W}_{i}$. Now select $\theta_{i} \in C^{\infty}(B, \mathbf{R})$ with $\theta_{i}>0$ on $\bar{U}_{i}$ and $\theta_{i}<0$ on $\partial W_{i}$.

We have $E_{\left.\right|_{W_{i}}} \simeq W_{i} \times X$ and $E_{\left.\right|_{W_{i}}}$ contains the sequence of mutually disjoint closed analytic subsets $W_{i} \times\left\{a_{j}\right\}, j \in J$. On $W_{i} \times\left\{a_{j}\right\}$ we consider the restriction of $\mu$ and perturb it with $\epsilon_{i j} \theta_{i} \circ \pi$. More precisely, we define near $\bar{W}_{i} \times\left\{a_{j}\right\}$

$$
\mu_{i j}=\mu+\epsilon_{i j} \theta_{i} \circ \pi
$$

where $\epsilon_{i j}>0$ are small enough constants to be chosen later. For every $x \in A$ we set $\mu_{1}(x)=\max \left\{\mu_{i j}(x) ;(i, j) \in H(x)\right\}$ where $H(x)=\{(i, j) \in$ $\left.I \times J ; x \in W_{i} \times\left\{a_{j}\right\}\right\}$. If $\epsilon_{i j}$ are small enough, then $\mu_{1}$ is continuous on $A$, $\mu_{1} \in \mathcal{B}\left(A, p^{*} \mathcal{M}\right)$, and $\mu \leq \mu_{1}<\mu+\eta$ on $A$. 
Moreover, on $\partial\left(W_{i} \times\left\{a_{j}\right\}\right)$ one has

$(*)$

$$
\mu_{1}>\mu_{i j}
$$

for every indices $(i, j) \in I \times J$.

We shall prove that $\mu_{1}$ has an extension $\widetilde{\mu_{1}}$ to a neighborhood $V$ of $A$ such that $\widetilde{\mu_{1}} \in \mathcal{B}\left(V, \pi^{*} \mathcal{M}\right)$ and this clearly will conclude the proof of in view of the approximation Proposition 1.

For this we choose open neighborhoods $D_{j} \subset \subset X$ of the points $a_{j}$ such that $\overline{D_{j}} \cap \overline{D_{j^{\prime}}}=\emptyset$ if $j \neq j^{\prime}$. The functions $\mu_{i j}$ defined on $W_{i} \times\left\{a_{j}\right\}$ can be extended to smooth functions $\widetilde{\mu_{i j}}$ on $W_{i} \times D_{j}$ which are 1-convex with respect to $\pi^{*} \mathcal{M}$. Indeed, if $p_{i j}^{\prime}$ and $p_{i j}^{\prime \prime}$ denote the projections of $W_{i} \times D_{j}$ on $W_{i} \times\left\{a_{j}\right\}$ and on $D_{j}$ respectively, then one may set

$$
\widetilde{\mu_{i j}}:=\mu_{i j} \circ p_{i j}^{\prime}+f_{\left.\right|_{D_{j}}} \circ p_{i j}^{\prime \prime}
$$

Put

$$
\Omega:=\bigcup_{(i, j) \in I \times J} W_{i} \times D_{j}
$$

and for $x \in \Omega, \widetilde{\mu_{1}}(x)=\sup \left\{\widetilde{\mu_{i j}}(x) ;(i, j) \in \Gamma(x)\right\}$ where $\Gamma(x)=\{(i, j) \in$ $\left.I \times J ; x \in W_{i} \times D_{j}\right\}$.

If $V \subset \Omega$ is a small enough open neighborhood of $A$, it follows then from $(*)$ that $\widetilde{\mu_{1}}$ is continuous on $V$ and in fact $\widetilde{\mu_{1}} \in \mathcal{B}\left(V, \pi^{*} \mathcal{M}\right)$, whence the proof of statement $(\boldsymbol{\beta})$.

We now go back to the proof of Theorem $1^{\prime}$. Since Theorem $1^{\prime}$ holds for $E^{\prime}$, there exists a smooth exhaustion function $\psi^{\prime}: E^{\prime} \rightarrow \mathbf{R}$ which is 1-convex with respect to $\left(\pi^{\prime}\right)^{*} \mathcal{M}$.

We fix some smooth function $\widetilde{\mu}>0$ defined near $\bar{V}$, where $V$ is a sufficiently small open neighborhood of $A$ such that $\widetilde{\mu}_{\left.\right|_{V}}$ is proper and $\widetilde{\mu}$ is 1-convex with respect to $\pi^{*} \mathcal{M}$ near $\bar{V}$.

By Proposition 4 there is a quasi-plurisubharmonic function $\beta: E \rightarrow$ $[-\infty, \infty)$ with $A=\{\beta=-\infty\}$. Also we may assume $\beta=0$ on $E \backslash V$. Then $\beta^{\prime}=\beta \circ \tau$ is quasi-plurisubharmonic on $E^{\prime}$ and $A^{\prime}:=\tau^{-1}(A)=\left\{\beta^{\prime}=-\infty\right\}$. Since $\psi^{\prime}$ is a smooth exhaustion function on $E^{\prime}$ which is 1-convex with respect to $\left(\pi^{\prime}\right)^{*} \mathcal{M}$, there is a strictly increasing smooth convex function $\delta:(0, \infty) \rightarrow(0, \infty)$ such that $\delta \circ \psi^{\prime}+\beta^{\prime}$ is 1 -convex with respect to $\left(\pi^{\prime}\right)^{*} \mathcal{M}$ on $E^{\prime} \backslash A^{\prime}$, and 


$$
\delta \circ \psi^{\prime}+\beta^{\prime}>\widetilde{\mu} \circ \tau
$$

on $\tau^{-1}(\partial V)$.

Now $E$ is covered by the open subsets $V_{1}:=V$ and $V_{2}:=E \backslash A$. On $V_{1}$ we consider the function $\varphi_{1}=\widetilde{\mu}$ and on $V_{2}$ the function $\varphi_{2}=\delta \circ \psi^{\prime} \circ \tau^{-1}+\beta$ and we define the function $\psi_{1}: E \rightarrow \mathbf{R}$ given by $\psi_{1}(x):=\max \left\{\varphi_{k}(x) ; k \in\right.$ $K(x)\}$, where $K=\{1,2\}$ and $K(x)=\left\{k \in K ; x \in V_{k}\right\}$.

Then $\psi_{1}$ is a continuous exhaustion function on $E$ and $\psi_{1} \in \mathcal{B}\left(E, \pi^{*} \mathcal{M}\right)$. Thus the proof of Theorem $1^{\prime}$ is complete.

\section{REFERENCES}

[1] A. Andreotti and H. Grauert, Théorèmes de finitude pour la cohomologie des espaces complexes, Bull. Soc. Math. France, 90 (1962), 193-259.

[2] E. Ballico, Coverings of complex spaces and q-completeness, Riv. Mat. Univ. Parma, (4), 7 (1981), 443-452.

[3] G. Coeuré and J.J. Loeb, A counterexample to the Serre problem with a bounded domain of $\mathbf{C}^{2}$ as fiber, Annals of Math., 122 (1985), 329-334.

[4] M. Colţoiu, Complete pluripolar sets, J. reine angew. Math., 412 (1992), 108-112.

[5] _ n-concavity of n-dimensional complex spaces, Math. Z., 210 (1992), 203-206.

[6] q-convexity. A survey., In: Complex analysis and geometry, Pitman Research Notes in Mathematics Series, 366 (1997), 83-93.

[7] J.-P. Demailly, Un example de fibré holomorphe non de Stein à fibre $\mathbf{C}^{2}$ ayant pour base le disque ou le plan, Invent. Math., 48 (1978), 293-302.

[8] C_ Cohomology of q-convex spaces in top degrees, Math. Z., 204 (1990), 283-295.

[9] A. Hirschowitz, Domaines de Stein et fonctions holomorphes bornées, Math. Ann., 213 (1975), 185-193.

[10] S. Kobayashi, Hyperbolic manifolds and holomorphic mappings, New York, Marcel Dekker, 1970.

[11] S. Lang, Introduction to complex hyperbolic spaces, Springer Verlag, 1987.

[12] P. Le Barz, A propos des revêtements ramifiés d'espace de Stein, Math. Ann., 222 (1976), 63-69.

[13] N. Mok, The Serre problem on Riemann surfaces, Math. Ann., 258 (1981), 145-168.

[14] R. Narasimhan, A note on Stein spaces and their normalizations, Ann. Sc. Norm. Sup. Pisa, 16 (1962), 327-333.

[15] M. Peternell, Algebraische Varietäten und q-vollständige komplexe Räume, Math. Z., 200 (1989), 547-581.

[16] H. Royden, Holomorphic fiber bundles with hyperbolic fiber, Proc. A.M.S., 43 (1974), 311-312.

[17] J.-P. Serre, Quelques problèmes globaux relatifs aux variétés de Stein, Colloque sur les fonctions de plusieurs variables, Bruxelles (1953), 53-68. 
[18] N. Sibony, Fibrés holomorphes et métrique de Carathéodory, C.R.A.S., 279 (1974), 261-264.

[19] Y.-T. Siu, All plane domains are Banach-Stein, Manuscripta math., 14 (1974), 101-105.

[20] Holomorphic fibre bundles whose fibers are bounded Stein domains with zero first Betti number, Math. Ann., 219 (1976), 171-192.

[21] H. Skoda, Fibrés holomorphes à base et à fibre de Stein, Invent. Math., 43 (1977), 97-107.

[22] J.-L. Stehlé, Fonctions plurisousharmoniques et convexité holomorphe dans certain fibrés analytiques, Lecture Notes in Math., 474 Sém. P. Lelong 1973/74, 155-180.

[23] K. Stein, Überlagerungen holomorph vollständiger komplexer Räume, Arch. Math., 7 (1956), 354-361.

[24] V. Vâjâitu, Approximation theorems and homology of q-Runge pairs in complex spaces, J. reine angew. Math., 449 (1994), 179-199.

[25] - Some convexity properties of proper morphisms of complex spaces, Math. Z., 217 (1994), 215-245.

[26] — One dimensional fibering over q-complete spaces, Nagoya Math. J., 151 (1998), 99-106.

Mihnea Colţoiu

Institute of Mathematics of the Romanian Academy

P.O. Box 1-764, RO 70700, Bucharest, Romania

mcoltoiu@stoilow.imar.ro

Viorel Vâjâitu

Institute of Mathematics of the Romanian Academy

P.O. Box 1-764, RO 70700, Bucharest, Romania

vvajaitu@stoilow.imar.ro 